\title{
La dynamique associative des Maliens de l'extérieur : enseignements tirés de deux dispositifs d'enquête originaux
}

Malian Migrants' Home Town Associations: Insights from Two Original Surveys La dinámica asociativa de los malienses del exterior: datos extraídos de dos dispositivos de encuesta originales

Claire Bernard, Lisa Chauvet, Flore Gubert, Marion Mercier et Sandrine Mesplé-Somps

\section{(2) OpenEdition}

\section{Édition électronique}

URL : https://journals.openedition.org/remi/7035

DOI : $10.4000 /$ remi. 7035

ISSN : $1777-5418$

Éditeur

Université de Poitiers

\section{Édition imprimée}

Date de publication : 1 décembre 2014

Pagination : 109-137

ISBN : 979-10-90426-23-8

ISSN : 0765-0752

Référence électronique

Claire Bernard, Lisa Chauvet, Flore Gubert, Marion Mercier et Sandrine Mesplé-Somps, «La dynamique associative des Maliens de l'extérieur : enseignements tirés de deux dispositifs d'enquête originaux », Revue européenne des migrations internationales [En ligne], vol. 30 - n³ et 4 | 2014, mis en ligne le 01 décembre 2017, consulté le 14 avril 2022. URL : http://journals.openedition.org/remi/7035 ; DOI : https://doi.org/10.4000/remi.7035 


\section{La dynamique associative des Maliens de l'extérieur : enseignements tirés de deux dispositifs d'enquête originaux}

\section{... Claire Bernard' ${ }^{1}$ Lisa Chauvet ${ }^{2}$, Flore Gubert ${ }^{3}$, Marion Mercier ${ }^{4}$ et Sandrine Mesplé-Somps ${ }^{5}$}

L'augmentation du nombre de migrants internationaux dans le monde s'est traduite par un intérêt croissant de la communauté scientifique pour la question des liens entre migration et développement des pays d'origine. Les économistes notamment se sont emparés de cette question à travers le prisme des transferts migratoires. Deux types de travaux en ont résulté : des travaux micro-économiques, examinant l'impact de la migration et des transferts sur les comportements des ménages récipiendaires (consommation et épargne, éducation, santé, etc.) et des travaux macro-économiques mesurant les retombées des transferts sur la croissance, la pauvreté ou d'autres indicateurs de développement à I'échelle des pays ${ }^{6}$.

Entre ces deux niveaux d'analyse, un niveau intermédiaire dit " méso-économique " reste toutefois peu investigué. En dehors des transferts à la famille, la littérature en sciences sociales nous apprend en effet que les migrants sont également souvent nombreux à participer au développement de leur localité

1 Économiste, Commissariat général à la stratégie et à la prospective, 18 rue de Martignac, 75007 Paris ; claire.bernard@strategie.gouv.fr

2 Chargée de recherche IRD, UMR DIAL, IRD-Université Paris Dauphine, 4 rue d'Enghien, 75010 Paris ; chauvet@ dial.prd.fr

3 Chargée de recherche IRD, UMR DIAL, IRD-Université Paris Dauphine et École d'Économie de Paris, 4 rue d'Enghien 75010 Paris ; gubert@ dial.prd.fr

4 Post-doctorante, ECARES, Université Libre de Bruxelles, Avenue F.D. Roosevelt 42, B1050 Bruxelles, Belgique ; mercier@dial.prd.fr

5 Chargée de recherche IRD, UMR DIAL, IRD-Université Paris Dauphine, 4 rue d'Enghien, 75010 Paris ; mesple@dial.prd.fr

6 Ce travail a bénéficié de financements de la Mairie de Paris (Programme Émergence), de I'AIRD et du ministère des Affaires étrangères français (FSP Mali contemporain). Nous remercions Mahamadou Dangnoko, Anda David et Nelly Rakoto-Tiana pour leurs contributions à la construction de la base de données sur les associations de migrants maliens de France, ainsi que Cecilia Navarra pour ses commentaires sur une version préliminaire de I'article. Nous remercions également vivement I'Institut National de la Statistique du Mali (INSTAT) pour sa collaboration dans la collecte des données au Mali. 
d'origine à travers des transferts collectifs dédiés au financement de projets de développement (projets d'infrastructure : écoles, dispensaires, magasins coopératifs, puits; projets productifs ; etc. $)^{7}$. Ces opérations sont généralement le fait $\mathrm{d}^{\prime}$ associations de migrants regroupant les ressortissants originaires d'un même village ou d'une même région. Si la dynamique associative des migrants a suscité l'intérêt de nombreux chercheurs anthropologues et géographes, elle est restée comparativement peu étudiée par les économistes en raison notamment d'un manque de données disponibles ${ }^{8}$. Pourtant, les conséquences de l'intervention de ces associations sur le développement des localités d'origine des migrants sont potentiellement importantes (Daum, 1995 ; Beauchemin et Schoumaker, 2009). Ce phénomène mérite donc d'être examiné d'un point de vue quantitatif afin de progresser dans la compréhension des liens entre migration et développement.

Le projet de recherche GLAMMS (Associations de migrants, Gouvernance et biens publics Locaux dans les pays d'origine : le cas des Associations de Migrants Maliennes et Sénégalaises en France et en Italie) s'inscrit dans la volonté d'améliorer l'état des connaissances sur le rôle des associations de migrants dans le développement des pays d'origine, en prenant pour cas d'étude le Mali et le Sénégal. Porté par une équipe de chercheurs de I'IRD et de Forum Internazionale ed Europeo di Ricerche sull'Immigrazione (FIERI, Italie), il repose sur une approche mêlant qualitatif et quantitatif et s'articule autour de deux grands axes : le premier vise à comprendre le mode d'organisation des associations de migrants en France et en Italie, les rapports qu'elles entretiennent avec les pouvoirs institutionnels et les acteurs associatifs ici et là-bas, leur mode d'intervention dans les communautés d'origine, et les changements, voire les ruptures, qu'elles y induisent; le second vise à analyser l'impact de l'action des associations de migrants sur la disponibilité en biens publics locaux dans les pays d'origine'.

Le projet a démarré par un travail important de production de données dont cet article vise à rendre compte. Deux bases de données originales, collectées respectivement en France et au Mali, ont en effet été constituées. La première, qui fait écho au travail réalisé par Daum en 1992 (Daum, 1993), rassemble l'ensemble des associations de ressortissants originaires du Mali déclarées au Journal Officiel en France. La seconde est issue d'une enquête représentative au niveau national réalisée en 2011 au Mali, dont un des modules a permis de recueillir, pour chaque commune rurale de l'échantillon, des informations sur le nombre d'associations créées par des ressortissants maliens en France et dans le reste du monde. À partir de ces deux bases de données, largement complémentaires, le présent article vise à dresser un panorama de la dynamique associative des Maliens de I'extérieur. À notre connaissance, de telles données

7 Voir notamment Daum (1998), Gauvrit et Le Bahers (2004), Lima (2005) et Tandian (2005) sur le Mali ; Kane (2001, 2002), Riccio (2007), Dia (2008) et Navarra et Salis (2011) sur le Sénégal ; mais aussi Iskander (2008) et Lacroix $(2005,2009)$ sur le Maroc et Simpser (2010) et Aparicio et Meseguer (2008) sur le Mexique.

8 Le travail mené sur les associations de migrants burkinabè par Beauchemin et Schoumaker (2009) est une exception en la matière.

9 Pour plus d'informations sur ce projet, le lecteur est invité à visiter la page web qui lui est dédiée : http://www.dial.ird.fr/projets-de-recherche/autres-projets/glamms. 
sur les associations de migrants, collectées à la fois " ici " et "là-bas ", n'ont encore jamais été produites. De plus, l'enquête réalisée au Mali permet d'évaluer le nombre de migrants internationaux originaires de chaque région du Mali et de mettre en regard la dynamique associative des Maliens de l'extérieur avec celle des migrations maliennes ${ }^{10}$.

Après une première section consacrée à une revue de la littérature sur les pratiques associatives des migrants sahéliens et plus particulièrement maliens, la deuxième section revient sur les sources de données mobilisées pour cette étude et les méthodes de collecte mises en œuvre. Nous présentons ensuite des statistiques descriptives sur les associations de migrants maliens en France et dans le reste du monde, en confrontant ces deux sources d'informations et en les comparant aux données sur l'émigration malienne. Enfin, la dernière section présente quelques-unes des analyses réalisées à partir de ces données et des pistes de recherche qu'elles ouvrent.

\section{Les pratiques associatives des migrants sahéliens : une revue de la littérature}

Les premiers travaux de recherche faisant état de la forte dynamique associative des migrants sahéliens, et de l'ampleur et de la diversité de leurs réalisations dans les localités d'origine, remontent aux années 1980 et 1990 (voir notamment Daum et al., 1988 ; Quiminal, 1991 ; Lavigne Delville, 1991 ; Institut Panos, 1993 ; Daum, 1995 et 1998 ; Blion et Verrière, 1998). Parmi les zones pourvoyeuses de migrants, c'est sur la région du bassin du fleuve Sénégal autrement appelée région des trois frontières, car située à cheval sur le Mali, le Sénégal et la Mauritanie - que s'est au départ focalisée l'attention en raison de son importante tradition migratoire et de sa forte dépendance aux transferts monétaires issus des migrations (Quiminal, 1993).

La structuration des migrants originaires de la vallée du fleuve Sénégal, majoritairement soninké, s'est d'abord faite à travers la constitution de caisses de solidarité villageoise (Quiminal, 1991 ; Daum, 1995 et 1998). Apparues dès les années 1960 dans les lieux d'installation des migrants, notamment dans les foyers situés en région parisienne ou dans des villes comme Rouen, ces caisses ont été mises en place pour subvenir aux besoins des membres de la communauté dans le pays d'accueil et pour assurer la subsistance des communautés villageoises dans le pays d'origine ${ }^{11}$. Au fur et à mesure de leur reconnaissance par les institutions internationales et les pouvoirs publics, elles se sont ensuite formalisées au point de devenir des acteurs à part entière de la solidarité internationale. En France, ce processus de formalisation a démarré à partir de 1981, lorsque les étrangers se sont vus accorder par l'État le droit de constituer des

10 L'enquête permet d'identifier les individus en migration et leur lieu de résidence. II est donc possible de calculer, pour chaque région du Mali, les effectifs de migrants par pays de destination et donc les taux de migration désagrégés à l'échelle régionale.

11 Comme I'ont souligné plusieurs auteurs (notamment Daum, 1998 et plus récemment Dia, 2008 et Gonin et Kotlok, 2009), participer à une caisse villageoise fait partie des obligations qui incombent aux migrants et auxquelles il leur est difficile de se soustraire. $C^{\prime}$ est en effet pour les notables restés au village, un moyen de contrôler les membres en migration. Gonin et Kotlok (2009) parlent à cet égard de " solidarité sous contrainte ". 
associations loi 1901. L'accès à ce statut formel a offert aux migrants une nouvelle visibilité et de nouveaux interlocuteurs tant dans les pays d'accueil (collectivités territoriales, ONG, etc.) que dans les pays d'origine (administrations régionales, voire nationales) et, partant, leur a donné accès à des financements extérieurs. Les caisses de solidarité sont ainsi devenues des associations puis des OSIM, Organisations de Solidarité Internationale de Migrants (Lacroix, 2006).

La toute première étude rendant compte de la diversité des interventions des migrants dans les villages de la région du fleuve Sénégal a été réalisée dans le cadre d'une action thématique programmée (ATP) du CNRS, et ses résultats ont été rendus publics en 1988 (Daum et al., 1988, cité par Gonin, 2001) 12. À partir d'enquêtes effectuées dans plus d'une vingtaine de foyers de la région parisienne et de Rouen et dans quarante-six villages du Mali et du Sénégal, cette étude s'est attachée à décrire le processus d'émergence et les conditions de réalisation des projets collectifs initiés par les migrants, ainsi que les modalités de leur gestion. Elle a également proposé une classification des projets réalisés qui montre que dans les années 1980, les migrants intervenaient déjà dans de nombreux domaines (coopératives d'achat, construction d'écoles, pharmacies villageoises, dispensaires, maternités, périmètres irrigués, etc.) (Gonin, 2001).

Dans le prolongement de ce travail, une enquête visant à dénombrer les projets portés par les migrants a été lancée (Institut Panos, 1992). En suivant la même approche " multi-située " que celle retenue dans le cadre du projet CNRS, cette enquête a été réalisée avec le souci de porter sur un échantillon aussi représentatif que possible des associations du fleuve Sénégal. Le travail a donc débuté par un recensement de ces associations à partir des archives du Journal Officiel $^{13}$ (Daum, 1993). Au total, 400 associations localisées en Île-de-France et en Seine-Maritime ont été identifiées. 105 d'entre elles ont ensuite été choisies et enquêtées, ainsi que les quatre-vingt-seize villages concernés par leurs actions. Au final, 346 projets initiés par ces associations ont été dénombrés, parmi lesquels 20,2 \% sont des projets dans le domaine de la santé (centres de santé), 19,9 \% dans le domaine de la consommation (centrales d'achat, magasins d'approvisionnement) et $17,9 \%$ dans le domaine de l'éducation (écoles). L'étude a par ailleurs permis de confirmer la forte dynamique associative des migrants originaires de la vallée du fleuve ainsi que son accélération à partir de 1987.

Le processus de décentralisation au Mali, initié dès 1992, donne un nouvel élan aux recherches consacrées aux pratiques associatives des migrants. Mais leur échelle d'analyse n'est plus la même. Les réformes nationales entreprises dans les trois pays frontaliers (en plus de la décentralisation malienne), et les spécificités des rapports entre associations de migrants et pouvoirs politiques dans chacun de ces pays, conduisent à distinguer les contextes nationaux et à ne plus considérer la région du fleuve Sénégal comme un tout homogène. Dans le cas des travaux portant sur le Mali, toutefois, I'attention continue d'être focalisée

12 Programme "La fonction émigrée dans les stratégies de développement ", sous la direction de Patrick Gonin, Paris, CNRS, Politiques et stratégies de développement dans le Tiers-monde, ATP/CNRS, E.N.S. Fontenay/Saint-Cloud, Centre de Géographie Rurale.

13 Seules les associations localisées en Île-de-France et en Seine-Maritime ont été incluses dans le recensement, ces deux régions regroupant $75 \%$ des migrants de la vallée du fleuve en 1992 (Daum, 1995). 
sur la seule région de Kayes, à l'ouest du pays. Plusieurs articles se sont notamment attachés à analyser dans quelle mesure la création des communes rurales et des conseils communaux consécutive au processus de décentralisation ${ }^{14}$ y avait modifié le mode opératoire des associations de migrants dont I'assise était au départ essentiellement villageoise (voir notamment Lima, 2005 et Quiminal, 2006). Comme I'explique Lima (2005), la décentralisation administrative a impliqué que les principaux domaines d'intervention des associations de migrants (santé, éducation, hydraulique), jusqu'alors dévolus à l'État central, sont devenus des prérogatives des communes nouvellement créées. Dotées d'une autonomie financière et budgétaire, ces dernières sont ainsi devenues les collectivités territoriales de référence du développement local. Par ailleurs, les villages qui étaient la cible des réalisations des associations ont perdu leur autonomie en devenant des composantes des communes rurales. Les projets visant à la fourniture de services et d'équipements publics au niveau des villages ont donc laissé place à des projets gérés à l'échelle des communes et figurant à ce titre dans les plans de développement communaux ou Programmes de Développement Économique, Social et Culturel (PDESC). L'ensemble de ces changements a eu des répercussions importantes sur le mode opératoire des associations. Avec la mise en place des conseils communaux, les migrants qui étaient jusque-là habitués à négocier avec les services administratifs régionaux ont notamment dû apprendre à interagir avec de nouveaux interlocuteurs. Ils ont également eu à s'adapter au changement d'échelle de leurs interventions, ce que reflète la multiplication des associations inter-villageoises, dont les limites ne recoupent toutefois pas toujours celles des territoires communaux ${ }^{15}$.

Comme le montre une étude rendue publique en 2004, ces changements institutionnels ont eu un impact sur les interventions des associations de migrants (Gauvrit et al., 2004). Prenant appui sur les résultats d'une enquête réalisée dans le cadre du Fonds de Solidarité Prioritaire (FSP) Codéveloppement Mali auprès de quarante-deux associations, trente et un villages et vingt et une communes de la région de Kayes ${ }^{16}$, cette étude montre d'abord que les associations agissent de moins en moins seules et que le nombre de projets cofinancés ne cesse d'augmenter ${ }^{17}$. En effet, l'analyse des 260 projets recensés

\footnotetext{
14 Avant la réforme de décentralisation, seules les communes urbaines avaient une entité administrative propre. Dorénavant, le territoire malien est découpé en 703 communes (rurales ou urbaines) réparties dans neuf régions (dont la capitale Bamako) et quarante-cinq cercles. Des conseils communaux sont élus tous les quatre ans depuis 1998/1999 et les communes disposent d'une autonomie financière et budgétaire.
}

15 Comme l'explique Lima (2005 : 300) à propos du cercle de Kayes, toutes les associations inter-villageoises n'ont pas attendu la décentralisation pour se constituer et certaines ont d'ailleurs joué un rôle indéniable dans le découpage communal. Dans d'autres cas, ce sont les ONG qui ont œuvré en faveur de ce changement d'échelle.

16 À la différence de I'enquête de I'Institut Panos, I'échantillon n'a pas été constitué dans le but d'être "représentatif " de la diversité des associations de la région, si bien qu'il n'est pas possible de généraliser les résultats obtenus.

17 La très forte progression du nombre de projets co-financés est également révélée par le programme Solidarité Eau (pS-Eau), un réseau d'acteurs de la coopération internationale intervenant dans les secteurs de l'eau, de I'assainissement et du développement. PS-Eau dispose d'une base de données régulièrement mise à jour qui dénombre les actions de développement menées dans le domaine de l'eau et de l'assainissement dans un grand nombre de pays. Ainsi, 121 actions ont été recensées au Mali par ce programme, dont certaines entreprises par ou en collaboration avec des migrants (chiffre tiré de http://www. pseau.org/outils/actions/action.php?pgmpseau_id=43\&l=fr, consulté le 10/02/2014). 
dans I'enquête fait ressortir que seuls $8 \%$ des projets mis en œuvre avant 1975 ont bénéficié du soutien d'un partenaire alors que cette proportion atteint $57 \%$ pour les projets mis en œuvre entre 1995 et 2004. Par ailleurs, les partenaires extérieurs et les bailleurs de fonds conditionnent souvent leur financement à la réalisation d'études de faisabilité, à l'implication plus systématique des populations bénéficiaires lors de la phase d'élaboration des projets ou encore à la préparation en amont des modalités de gestion des réalisations. Ces conditions tendent à favoriser une meilleure prise en charge des réalisations par les populations locales et donc à mieux garantir la pérennité des projets financés par les migrants.

II ressort de cette littérature deux éléments remarquables à propos du rapport que les migrants originaires de la vallée du fleuve Sénégal et notamment de la région de Kayes entretiennent avec leurs villages ou leurs régions d'origine. Le premier est son caractère extrêmement organisé et structuré ; le second est son inscription dans le temps. Comme le soulignait déjà Daum à la fin des années 1990, la pérennité des associations de migrants et leur capacité à s'adapter aux transformations nombreuses qui ont eu cours depuis leur création tant en France qu'au Mali tranchent en effet avec le caractère souvent instable et ponctuel de ce type d'organisation relevé par la sociologie des associations dans I'espace français (Daum, 1998 : 117). La question qui reste en suspens, toutefois, est celle de l'impact de ces associations. En effet, bien que ces dernières soient de plus en plus reconnues comme des acteurs du développement, aucune étude quantitative n'a été réalisée pour évaluer les retombées de leur intervention dans une perspective nationale, comme cela a par exemple été fait au Burkina Faso (Beauchemin et Schoumaker, 2009). Cet état de fait tient à un manque criant de données actualisées tant sur le nombre d'associations de migrants menant des actions de développement local au Mali, qu'elles soient localisées en France ou dans un autre pays d'accueil, que sur le nombre et le type de réalisations de ces associations dans les villages d'origine. Le seul travail d'envergure est celui réalisé par Daum en 1992, mais il n'a malheureusement pas été actualisé alors même que la dynamique associative n'a cessé de s'intensifier et d'évoluer. En outre, n'ont été recensées que les associations localisées en Île-de-France et en Seine-Maritime et constituées par des migrants originaires de la région du fleuve Sénégal. Une analyse focalisée sur le Mali nécessiterait d'exclure de ce recensement les associations sénégalaises et mauritaniennes et de lui ajouter toutes les associations créées par des ressortissants maliens originaires d'autres régions du Mali. Car ces derniers font eux aussi preuve d'une forte propension à se regrouper en vue d'impulser des projets de développement dans leurs localités d'origine, même s'ils sont en proportion moins nombreux à émigrer vers l'étranger (cf. Tableau 7 en annexe $B$ ). Cette réalité justifie que le phénomène associatif malien soit étudié dans son ensemble et non plus uniquement sur la région de Kayes, afin de mettre en perspective les spécificités régionales, d'identifier des tendances nationales et de rendre compte de l'évolution globale des pratiques associatives des migrants maliens, que ces derniers résident en France ou dans un autre pays étranger ${ }^{18}$. C'est l'objectif des deux dispositifs mis

18 L'approche comparative entre différentes régions du Mali a déjà été adoptée pour I'analyse des rapports entre les migrants et leur communauté d'origine (Daum et Cissé, 2009), mais à notre connaissance jamais concernant spécifiquement les associations de migrants. 
en œuvre dans le cadre du projet GLAMMS dont une présentation détaillée fait l'objet de la section suivante.

\section{Deux dispositifs innovants de collecte de données sur les associations de migrants maliens}

\section{Le recensement des associations de Maliens en France}

Le premier dispositif que nous avons mis en œuvre a consisté à réaliser un recensement aussi large que possible des associations formelles de migrants maliens en France. Le phénomène associatif au sens large est un objet d'étude par nature complexe, puisqu'il peut reposer sur l'informalité des relations entre les membres, ce qui rend difficile l'identification de toutes les associations créées sur le territoire français. Cependant, pour avoir une existence légale et une personnalité juridique en France, les associations doivent, selon la loi du $1^{\text {er }}$ juillet 1901, être déclarées en préfecture ou en sous-préfecture. Cette démarche simple donne lieu à une déclaration de création d'association dans le Journal Officiel.

À la manière de Daum (1992), nous nous sommes appuyées sur les archives du Journal Officiel. Cependant, notre travail se distingue du sien sur deux points. D'une part, notre recensement couvre tout le territoire français, alors que le sien était restreint à l'Île-de-France et à la Seine-Maritime. D'autre part, Daum a eu recours à une typologie distinguant les associations de la vallée du fleuve des autres associations d'Afrique alors que nous avons choisi de faire porter notre recensement sur l'ensemble du Mali. Cette stratégie nous permet de dresser un panorama comparatif des pratiques associatives des migrants en fonction de leur région d'origine que nous discutons en troisième section de cet article. In fine, nos données confirment la forte concentration géographique des associations en île-de-France : 78 \% d'entre elles y ont leur siège social (en particulier, $27 \%$ en Seine-Saint-Denis et $22 \%$ à Paris). En revanche, moins de $1 \%$ des associations que nous avons identifiées sont localisées en Seine-Maritime. Nos données confirment également la prédominance des associations ciblées sur la région Kayes, mais montrent que les migrants originaires d'autres régions du Mali participent eux aussi activement à la création d'associations de développement. Nous présentons ci-dessous plus en détail la méthodologie suivie pour collecter les données sur les associations maliennes en France.

\section{Méthodologie}

Pour recenser les associations formelles de ressortissants maliens en France, nous avons eu recours aux archives du Journal Officiel depuis 1981. Ces archives sont disponibles en ligne sur le site du Journal Officiel ${ }^{19}$ qui permet de rechercher une déclaration de création ou de modification d'association à partir de son nom. Elles sont également disponibles à partir du site www.refasso.com, qui propose une base de données quasi exhaustive des associations françaises. Cet annuaire, réalisé à partir des publications au Journal Officiel, regroupe plus de 2100000 associations actives ou dissoutes, et permet en particulier d'effectuer des recherches par mot-clé.

19 http://www.journal-officiel.gouv.fr/ 
Pour réaliser le recensement des associations de migrants maliens en France, nous avons d'abord effectué des recherches par mot-clé sur le site www.refasso. com. Cet outil permet d'identifier toutes les associations comportant, soit dans leur titre soit dans leur objet, un des mots suivant: Mali, malien, malienne, maliens, maliennes. Dans une seconde étape, nous avons affiné la recherche en utilisant comme mots-clés les noms des quelque 10000 villages maliens listés par le Recensement Général de la Population et de I'Habitat de 1998 (INSTAT). Toutes les associations retrouvées par le biais de ces mots-clés ont ensuite été vérifiées une à une, afin de nous assurer, d'une part, qu'il s'agit d'associations de migrants (et non pas, par exemple, d'associations caritatives créées à l'initiative d'individus n'étant pas originaires du Mali) et d'autre part, qu'elles interviennent bien au Mali. En effet, bon nombre de villages maliens portent le même nom que des villages d'autres pays d'Afrique de l'Ouest. Nous avons donc recherché dans les archives du Journal Officiel, ainsi que dans toutes les autres sources disponibles en ligne (sites Internet des associations, des collectivités territoriales, etc.), des informations complémentaires sur la zone d'activité de chacune de ces associations, afin de ne conserver que celles intervenant au Mali. Lorsque plusieurs villages/communes malien(ne)s dans des cercles ou des régions différents portaient le même nom, un travail de recherche en ligne supplémentaire a été entrepris afin de déterminer précisément la zone d'intervention de l'association.

Toutes les informations disponibles sur les associations identifiées lors de ces deux étapes ont ensuite été codées dans une base de données harmonisée. Pour chacune des associations recensées, nous avons saisi son titre, son objet, sa date de création et le code postal de son siège social. Lorsque l'information était disponible, nous avons également conservé l'adresse de son siège social, un numéro de téléphone et une adresse e-mail. Les associations recensées ont ensuite été géoréférencées selon leur zone d'intervention sur le territoire malien, sur la base du découpage territorial proposé par le Recensement Général de la Population et de I'Habitat (INSTAT, 1998). Deux informations ont été codées : le niveau d'intervention de l'association (localité - village ou quartier -, commune, cercle ou région); le nom et le code ${ }^{20}$ de la zone d'intervention.

À l'issue de ces trois étapes (recherche par mot-clé, constitution de la base et géolocalisation), la base de données finale regroupe 565 associations maliennes déclarées au Journal Officiel français entre 1981 et 2011. Parmi ces 565 associations, 364 ciblent leur activité sur un village ou un quartier ${ }^{21}, 131$ sur une commune et soixante-dix sur un cercle ou une région du Mali.

Le tableau 1 présente la répartition de ces 565 associations selon le terrain où elles ont déclaré intervenir au Mali. Quelle que soit la région considérée, les associations ciblées sur un village apparaissent largement majoritaires, et la plupart d'entre elles interviennent dans des localités rurales. La distribution par région de ces associations sera commentée dans la troisième partie.

20 Codes des régions, cercles, communes et localités issus du Recensement Général de la Population et de I'Habitat (INSTAT, 1998).

21 Les communes rurales sont divisées en villages (en moyenne, quinze villages par commune) et les communes urbaines en quartiers. 
Tableau 1 : Échantillon des associations recensées dans le Journal Officiel français et répartition par région malienne (1981-2011)

\begin{tabular}{|c|c|c|c|c|c|c|}
\hline & $\begin{array}{c}\text { Nombre } \\
\text { d'associations }\end{array}$ & $\begin{array}{r}\mathrm{N} \\
\text { d'ass } \\
\text { ciblé } \\
\text { lo }\end{array}$ & $\begin{array}{l}\text { nbre } \\
\text { siations } \\
\text { sur une } \\
\text { lité* }\end{array}$ & $\begin{array}{r}N \\
\text { d'ass } \\
\text { ciblé } \\
\text { co }\end{array}$ & $\begin{array}{l}\text { nbre } \\
\text { ciations } \\
\text { sur une } \\
\text { mune }\end{array}$ & $\begin{array}{l}\text { Nombre } \\
\text { d'associations } \\
\text { ciblées sur un } \\
\text { cercle ou une }\end{array}$ \\
\hline & & Total & Rural** & Total & Rural** & \\
\hline Mali & 565 & 364 & 322 & 131 & 109 & 70 \\
\hline Kayes & 385 & 277 & 245 & 77 & 68 & 31 \\
\hline Koulikoro & 67 & 34 & 30 & 22 & 19 & 11 \\
\hline Sikasso & 16 & 10 & 9 & 6 & 5 & 0 \\
\hline Ségou & 14 & 6 & 6 & 1 & 1 & 7 \\
\hline Mopti & 57 & 32 & 28 & 17 & 10 & 8 \\
\hline Tombouctou & 9 & 1 & 1 & 4 & 4 & 4 \\
\hline Gao & 5 & 1 & 1 & 3 & 2 & 1 \\
\hline Kidal & 3 & 3 & 2 & 0 & 0 & 0 \\
\hline Bamako & 9 & 0 & 0 & 1 & 0 & 8 \\
\hline
\end{tabular}

* Le terme " localité " fait référence aux villages dans le cas des communes rurales et aux quartiers dans le cas des communes urbaines.

** Le Mali est composé de plus de 10000 villages et quartiers regroupés dans 703 communes. Une trentaine de ces communes a un statut urbain tandis que les autres sont dites rurales. Les communes urbaines sont constituées de quartiers, mais aussi de villages proches des agglomérations urbaines qui appartiennent à l'entité administrative " commune urbaine ".

Source : Calculs des auteurs à partir des déclarations des associations de migrants maliens au Journal Officiel français, compilées sur www.refasso.com.

\section{Les limites du dispositif}

Une première difficulté inhérente à la volonté de recenser des associations tient bien sûr au caractère parfois informel des pratiques associatives. De fait, nous ne sommes en mesure d'identifier ici que les associations déclarées en préfecture ou en sous-préfecture. Cependant, la démarche de déclaration d'une création d'association en préfecture ou en sous-préfecture est relativement simple et rapide. Elle donne en outre accès à un statut formel rendant possible certaines activités (demande de subventions publiques, ventes, etc.) et à de potentiels avantages (déductions fiscales, etc.). Au regard des opportunités que I'inscription offre, il est probable qu'une part importante des associations actives soit déclarée au Journal Officiel.

Deuxièmement, bien que la méthode de recherche par mot-clé permette de retrouver une grande partie des associations de Maliens déclarées en France, il n'est pas exclu que certaines associations de ressortissants, quand bien même elles ont été déclarées au Journal Officiel, n'apparaissent pas dans notre recensement. C'est le cas si elles ne comportent ni dans leur titre, ni dans leur objet, I'un des mots-clés précédemment cités. Cependant, l'élargissement de la recherche à tous les dérivés du nom "Mali ", ainsi qu'à tous les noms de villages maliens recensés en 1998, et l'application de ces mots-clés non seulement aux titres, mais aussi aux objets des associations, nous permet de minimiser ce risque. À l'inverse, il est possible que certaines associations de solidarité à destination 
du Mali dirigées par des non-Maliens soient incluses dans notre recensement à tort. Une vérification systématique des objets déclarés par ces associations nous autorise toutefois à penser que nous avons réduit ce biais autant que possible.

Enfin, une troisième limite doit être considérée : elle tient à la question des dissolutions d'associations. Tout comme la procédure de création, la procédure de dissolution d'association est simple et rapide. Cependant, la déclaration officielle d'une dissolution d'association n'est pas automatique en pratique : une association peut très bien ne plus être active, sans que cela ne soit déclaré. Dès lors, il est possible que notre base de données comprenne des associations aujourd'hui inactives ${ }^{22}$. De ce fait, nous présentons dans la suite du document les associations par période d'inscription au Journal Officiel, afin d'avoir une idée de la dynamique associative des migrants maliens de France.

\section{L'enquête EMOP}

Pour compléter ce panorama, nous le confrontons à une seconde source: les données collectées auprès d'un large échantillon de communes du Mali. L'Institut National de la Statistique (INSTAT) du Mali a mis en place, en 2011, une Enquête Modulaire et Permanente auprès des Ménages (EMOP). II s'agit d'une enquête par sondage, stratifiée à deux degrés, sur un échantillon de 7176 ménages représentatif au niveau national et au niveau des zones urbaines et rurales des neuf régions du Mali. Cette enquête est réalisée en quatre passages, chaque passage se déroulant sur trois mois. Le questionnaire de I'EMOP comporte un module de base ciblant les ménages et les membres qui les composent et incluant des sections sur les caractéristiques démographiques, l'éducation, la santé et la consommation des personnes interrogées. Lors du deuxième passage de cette enquête réalisé entre juillet et septembre 2011, nous avons, en partenariat avec I'INSTAT, conçu et ajouté à ce module de base un module Commune portant sur les caractéristiques des 371 communes rurales tirées de manière aléatoire à partir du plan de sondage. N'enquêter que des communes rurales a été un choix délibéré de notre part. En effet, il nous est apparu qu'en milieu rural il serait plus facile d'identifier les personnes les mieux informées pour répondre aux questions posées dans ce module. En outre, les informations à recueillir sont comparativement moins nombreuses en milieu rural, les acteurs publics locaux étant moins nombreux et les structures communales moins complexes. Le questionnaire a été administré par des enquêteurs de I'INSTAT formés par les cadres de I'Institut et nous-mêmes. Les enquêteurs devaient recueillir un large éventail d'informations sur la commune en s'adressant aux personnes les plus à même de fournir les renseignements : en priorité, le maire et le secrétaire de mairie, mais aussi, si nécessaire, le régisseur, les adjoints ou d'autres conseillers municipaux. Une limite inhérente à cette manière de procéder tient bien sûr à la nature déclarative des informations recueillies. L'administration des questionnaires à plusieurs représentants d'une même collectivité permet cependant de limiter ce biais.

22 Sur la période récente (fin des années 2000), il est également possible que certaines associations aient été déclarées sans avoir d'existence réelle, ces dernières n'étant qu'une façade juridique créée pour permettre d'influer sur l'issue d'un vote organisé en 2009 et visant à élire les représentants des Conseils de base des Maliens de l'extérieur au sein du bureau du Haut Conseil des Maliens de l'extérieur à Bamako. Le vote était en effet fondé sur le principe $\mathrm{d}^{\prime}$ " une association, une voix" 
Le module Commune de l'enquête EMOP contient les informations suivantes concernant les associations de migrants : existence ou non d'une association de ressortissants active au sein de la commune (que celle-ci cible la commune dans son ensemble ou un ou plusieurs des villages qui la composent) et nombre d'associations de ressortissants actives basées dans d'autres localités maliennes, en France et dans le reste du monde. Ces données nous permettent de dresser un panorama des pratiques associatives en milieu rural des migrants maliens en 2011, par région du Mali et par zone d'émigration ${ }^{23}$. On dénombre ainsi plus de 2000 associations de ressortissants, dont 794 sont localisées en dehors des frontières maliennes ${ }^{24}$. La répartition de ces associations par région est présentée dans le tableau 2.

Tableau 2 : Répartition par région du Mali des associations de migrants en milieu rural

\begin{tabular}{|c|c|c|c|c|c|}
\hline & \multirow{2}{*}{$\begin{array}{l}\text { Nombre de communes } \\
\text { rurales enquêtées }\end{array}$} & \multicolumn{2}{|c|}{$\begin{array}{c}\text { Associations de } \\
\text { France* }\end{array}$} & \multicolumn{2}{|c|}{$\begin{array}{c}\text { Associations du reste } \\
\text { du monde* }\end{array}$} \\
\hline & & Effectif** & $\%$ & Effectif & $\%$ \\
\hline Mali & 371 & 307 & 100 & \begin{tabular}{|l|}
487 \\
\end{tabular} & 100 \\
\hline Kayes & 62 & 176 & 57,3 & 102 & 20,9 \\
\hline Koulikoro & 50 & 87 & 28,3 & 127 & 26,1 \\
\hline Sikasso & 65 & 14 & 4,6 & 72 & 14,8 \\
\hline Ségou & 77 & 14 & 4,6 & 27 & 5,5 \\
\hline Mopti & 75 & 15 & 4,9 & 133 & 27,3 \\
\hline Tombouctou & 25 & 0 & 0 & 11 & 2,3 \\
\hline Gao & 12 & 1 & 0,03 & 15 & 3,1 \\
\hline Kidal & 5 & 0 & 0 & 0 & 0 \\
\hline
\end{tabular}

* Les chiffres présentés dans ce tableau sont issus des données de l'enquête EMOP (modules GLAMMS, 2011, INSTAT IRD), pondérées afin de corriger pour l'attrition dans l'échantillon. Le système de pondération adopté est présenté en annexe A. ** Le nombre total d'associations de France identifiées grâce à l'enquête EMOP ne peut être directement comparé au nombre d'associations recensées via le Journal Officiel français puisqu'il correspond à un échantillon de communes maliennes et non au pays entier. II est aussi possible que certaines associations comptabilisées dans le recensement soient, en 2011, dissoutes.

Source : Calculs des auteurs à partir des données pondérées de l'enquête EMOP, modules GLAMMS, 2011, INSTAT IRD.

Il importe ici de noter que deux autres modules ont été ajoutés lors de ce deuxième passage de l'enquête EMOP : un module Migration, appliqué aux ménages, et un module Village ${ }^{25}$. Les renseignements concernant les 597 villages enquêtés ont été recueillis auprès des chefs de village. En particulier, des

23 Le module Commune a aussi permis de collecter des informations sur la composition et le fonctionnement des conseils municipaux ainsi que sur les projets de développement initiés dans la commune depuis 2004.

24 Les chiffres issus de l'enquête EMOP sont pondérés afin de corriger pour l'attrition dans l'échantillon. Le système de pondération adopté est présenté en annexe A.

25 Dans le reste du document et dans les tableaux ci-dessous présentés, ces trois modules supplémentaires (Commune, Village et Migration) sont aussi nommés modules GLAMMS, du nom du projet de recherche qui a été à l'origine de ce travail de collecte de données. 
informations sur la disponibilité en biens publics locaux (type, date de création, mode de financement dont financement des associations de migrants, etc.) ont été collectées ${ }^{26}$.

La section suivante présente quelques statistiques descriptives sur le phénomène associatif des Maliens de France élaborées à partir de ces deux bases de données.

\section{Panorama des associations de Maliens de France}

Les Maliens migrant en France proviennent traditionnellement de la région de Kayes, à l'ouest du Mali. La tradition migratoire malienne a été nourrie au fil des siècles par la croissance démographique, le commerce, les guerres et les chocs climatiques. Dans la région de Kayes, frontalière avec la Sénégambie, les migrations saisonnières, suivant le rythme de la culture et du commerce du coton et de l'arachide, ont marqué l'évolution des pratiques migratoires à la période coloniale, alors que la construction d'infrastructures de transport amplifiait le développement de ces migrations circulaires dans la sous-région. En parallèle, le recrutement dans la marine marchande et dans l'armée de Maliens, et en particulier de Soninké originaires de la région de Kayes, puis l'appel de la France aux jeunes travailleurs africains au moment de la reconstruction, ont stimulé la migration vers la métropole. De circulaires et saisonnières, ces migrations ont progressivement donné naissance à des migrations d'installation émanant largement de la région de Kayes.

Sur les 56500 migrants maliens en France dénombrés par le module Migration de I'enquête EMOP 2011, 60,3 \% proviennent de cette région, alors qu'elle représente moins de $15 \%$ de la population du pays (cf. Tableau 7 en annexe B). La surreprésentation des ressortissants de la région de Kayes parmi les Maliens de France se retrouve dans notre recensement des associations de ressortissants maliens déclarées au Journal Officiel : 385 (soit 68 \%) d'entre elles ciblent la région de Kayes. Au sein des associations intervenant dans cette région, on trouve une représentation plus importante des cercles de Kayes et de Yelimané. Parmi les 371 associations de la région de Kayes pour lesquelles le cercle d'intervention est connu ${ }^{27}, 38,3 \%$ interviennent dans le cercle de Kayes et $23,3 \%$ dans le cercle de Yelimané. Le phénomène associatif des Maliens de France reflète donc de manière particulièrement claire l'importance de la région de Kayes, et notamment des cercles de Kayes et de Yelimané, dans les flux de migrations dirigés vers la France ${ }^{28}$.

Les associations de migrants recensées au Journal Officiel français et ciblant les autres régions du Mali sont principalement concentrées sur les régions de Koulikoro et de Mopti, qui regroupent respectivement $11,8 \%$ et $10 \%$ des

26 Le module Village contient aussi des questions sur le fonctionnement des conseils villageois et le mode de gestion des structures publiques telles que les centres de santé et les points d'eau.

27 Quatorze associations ciblent la région de Kayes dans son ensemble.

28 Selon I'EMOP, 61 \% des migrants maliens en provenance de la région de Kayes et résidant en France proviennent du cercle de Kayes ; $16 \%$ du cercle de Yelimané. 
associations. D'après l'enquête EMOP 2011, ces deux régions comptent respectivement $15,2 \%$ et $0,6 \%$ des émigrés en France (cf. Tableau 7 en annexe B). Le phénomène associatif des migrants en France originaires de Koulikoro apparait donc cohérent avec l'importance de la région dans les flux d'émigration maliens. En revanche, l'activité associative des Maliens de France originaires de la région de Mopti apparaît très importante par rapport au poids de cette région dans les flux de départ, ce qui à notre connaissance n'avait jamais été identifié dans la littérature sur la dynamique associative malienne. Finalement, relativement peu $\mathrm{d}^{\prime}$ associations de Maliens recensées en France interviennent sur les cinq autres régions maliennes : Sikasso (2,8 \%), Ségou (2,5\%), Tombouctou (1,5\%), Gao et Kidal (moins de $1 \%$ ). Ces cinq régions sont aussi relativement peu concernées par la migration vers la France. Seulement 6,3\% des Maliens de France sont originaires de la région de Ségou, 2,2 \% de la région de Sikasso et moins de $0,3 \%$ des trois régions du Nord (Tombouctou, Gao, Kidal) (cf. Tableau 7 en annexe B). Enfin, la situation de Bamako apparait particulière : cette ville-région compte en effet $15,2 \%$ des émigrés vers la France d'après le module Migration de l'EMOP 2011, alors qu'elle ne représente que 1,5\% des associations de ressortissants identifiées en France. Cela suggère que la dynamique associative des migrants maliens de France reste centrée sur le milieu rural malgré le processus d'urbanisation du pays.

Notre recensement des associations de migrants maliens sur le territoire français semble donc refléter de façon cohérente les chiffres sur l'émigration tirés de l'enquête EMOP de 2011 : nous observons une forte dynamique associative des Maliens originaires de la région de Kayes, et dans une moindre mesure, de la région de Koulikoro. En revanche, deux différences apparaissent entre la répartition des associations sur le territoire malien et les statistiques migratoires apportées par l'enquête EMOP : une dynamique associative forte de la région de Mopti, par rapport à son poids dans les flux de migration, et à l'inverse relativement faible pour Bamako. En distinguant les localités d'intervention des associations par milieu (urbain ou rural), la dynamique associative des migrants maliens apparaît plus importante en milieu rural : 76,3 \% des associations recensées au Journal Officiel ciblent explicitement un village ou une commune rurale, alors que $65 \%$ de la population malienne ${ }^{29}$ est rurale. La répartition par région des associations de migrants identifiées par le recensement en France reste semblable si I'on se concentre sur les associations actives dans des zones rurales. Ainsi, 72,6 \% des associations identifiées qui agissent dans le monde rural sont ciblées sur Kayes. Ensuite, 11,4 \% d'entre elles interviennent à Koulikoro et 8,8 \% à Mopti. Les régions de Sikasso, Ségou, Tombouctou, Gao et Kidal sont ici encore assez peu concernées (cf. Tableaux 1 et 3).

Ces statistiques tirées du recensement des associations en France, et notamment de celles intervenant en zone rurale, peuvent être confrontées aux informations sur les associations de France contenues dans le module Commune de l'enquête EMOP, administré aux représentants de 371 communes rurales. On dénombre dans l'échantillon EMOP plus de 2000 associations de migrants (internationaux ou internes). Parmi elles, 794 sont localisées à l'exté-

29 Source : RGPH 2009, INSTAT. 
rieur du Mali, dont 307 en France ${ }^{30}$. La France rassemble donc environ $39 \%$ des associations de Maliens de l'extérieur identifiées dans cette enquête. Cela suggère une dynamique associative forte des Maliens de France par rapport au poids qu'ils représentent dans les flux migratoires. En effet, d'après le module Migration de l'enquête EMOP 2011, 17,2 \% des Maliens en migration à l'étranger au moment de l'enquête vivent dans I'hexagone.

La distribution de ces associations sur le territoire malien en milieu rural fait là encore ressortir la spécificité de la région de Kayes en cohérence avec les caractéristiques de la migration malienne. Ainsi, sur les 307 associations identifiées par l'enquête EMOP basées en France, 176 interviennent dans la région de Kayes, qui compte de loin le plus grand nombre d'associations de migrants françaises. Suivent les régions de Koulikoro (quare-vingt-sept associations), Mopti, Ségou et Sikasso (respectivement quinze, quatorze et quatorze associations). D'après l'enquête EMOP, Gao, Tombouctou et Kidal comptent très peu, ou pas du tout, d'associations de migrants en France.

À partir de ces chiffres, on peut estimer la répartition des associations de France sur I'ensemble du territoire rural malien (cf. Tableau 3 ) : 57,3 \% des associations de France interviennent dans la région de Kayes et $28,3 \%$ dans la région de Koulikoro. Viennent ensuite les régions de Mopti, Ségou et Sikasso avec respectivement $4,9 \%, 4,6 \%$ et $4,6 \%$ des associations, les trois régions du Nord représentant chacune moins de $1 \%$ du total. Les données issues de l'enquête EMOP reflètent donc une répartition par région des associations de migrants en France relativement similaire à celle obtenue à partir du recensement des associations déclarées au Journal Officiel français. Cependant, l'importance respective de ces trois régions diffère d'une source à l'autre (Figure 1) : I'EMOP comptabilise plus d'associations à Koulikoro que le Journal Officiel, alors qu'inversement l'activité associative dans les régions de Kayes et de Mopti y est estimée plus faible. Plusieurs éléments d'explication peuvent être avancés. En particulier, le recensement via le Journal Officiel est susceptible d'inclure des associations dissoutes en 2011, ou qui ont été déclarées dans un but électoral, mais qui, de fait, sont fictives. Inversement, il est possible que nous identifiions dans I'enquête EMOP des associations actives en pratique, mais non déclarées au Journal Officiel français.

Les deux sources de données mobilisées ici nous offrent un panorama riche de la dynamique associative des migrants maliens de France. Grâce aux informations disponibles sur la date de déclaration des associations, quelques éléments factuels sur la chronologie de ce phénomène associatif peuvent être ajoutés (Tableau 3 et Figure 2). Ils ne sont que parcellaires puisque nous ne pouvons pas identifier les associations dissoutes. De plus, la formalisation des regroupements en associations et leur déclaration en préfecture, ou en souspréfecture, sont un phénomène qui s'est développé au cours des dernières décennies bien que certaines associations aient commencé à agir dans leurs communautés d'origine bien avant leur déclaration au Journal Officiel (Gauvrit et al., 2004). Notre base de données ne nous permet malheureusement pas de connaître le temps qui s'est écoulé entre la date de création de chaque asso-

30 Les chiffres issus de l'enquête EMOP sont pondérés afin de corriger pour l'attrition dans l'échantillon. Le système de pondération adopté est présenté en annexe A. 


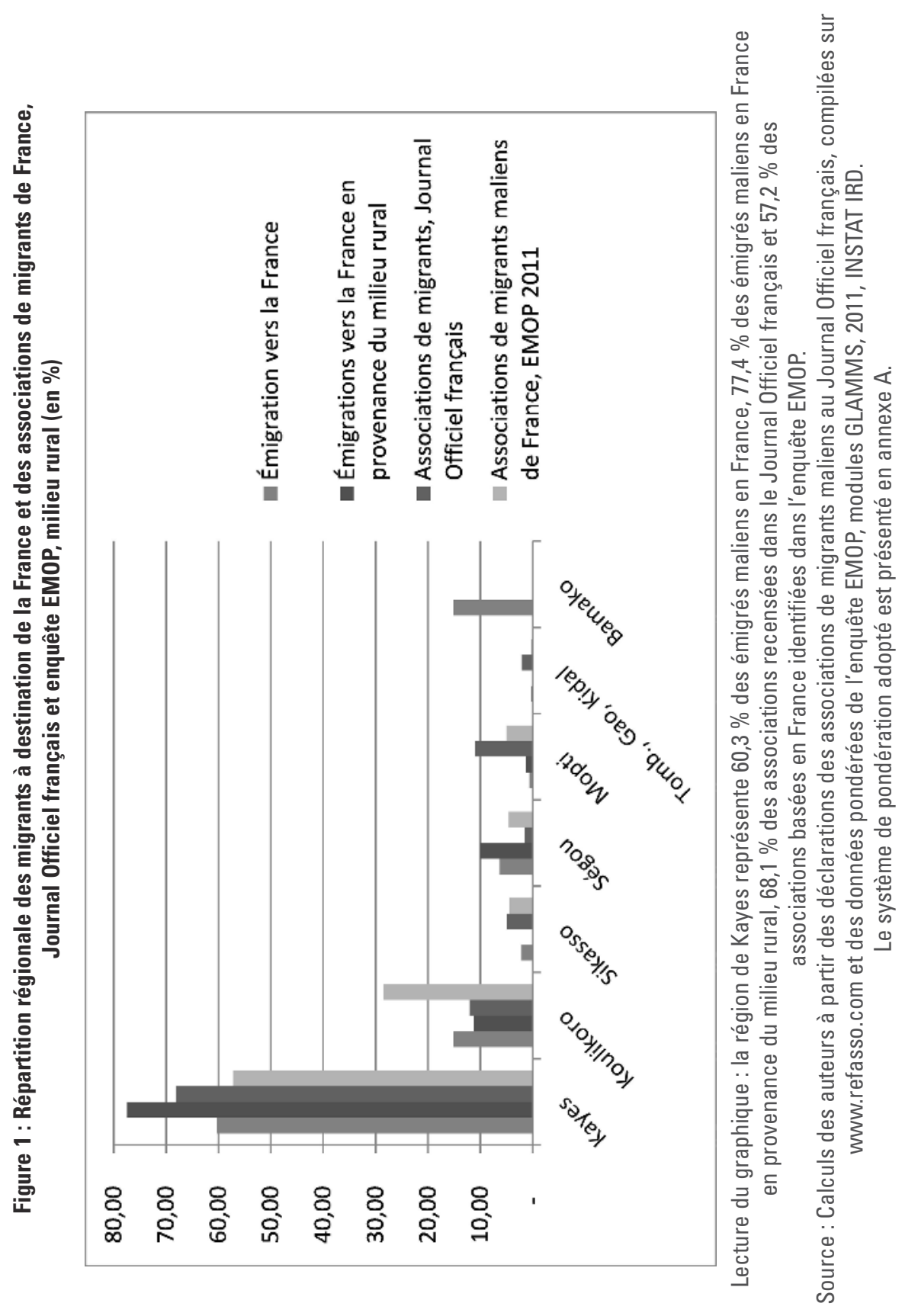


ciation et sa date de déclaration. Ces considérations faites, nous observons cependant que le phénomène associatif en France est ancien. En effet, 20,5 \% des associations recensées (116) ont été déclarées entre 1981 et 1995 . Une nette accélération se dégage dans les années 2000 : ainsi, soixante-cinq associations ont vu le jour entre 1995 et 1999, 140 entre les années 2000 et 2004 et 244 après 2005. Au final, $68 \%$ de notre échantillon est constitué d'associations déclarées depuis I'an 2000. Par ailleurs, la répartition des associations entre les différentes régions maliennes est restée relativement stable depuis le début de la période et il en est de même si l'on se concentre sur les zones rurales (Tableau 3). Ce dynamisme récent du mouvement associatif est peut-être en partie lié au mode de désignation retenu pour élire, en 2009, les représentants des Conseils de base des Maliens de l'extérieur au sein du bureau du Haut Conseil des Maliens de I'extérieur à Bamako. Certaines des associations déclarées à cette période pourraient ne pas avoir de réelles activités. II est possible aussi que ce dynamisme reflète une vague de formalisation de regroupements informels préexistants plutôt qu'une vague de création d'associations. Enfin, le développement de modes de financement spécifiquement adressés aux associations, tels que les projets de co-développement, est un autre élément potentiel d'explication à cette tendance.

\section{Panorama des associations de Maliens du reste du monde}

L'enquête EMOP apporte un éclairage supplémentaire sur les pratiques associatives des ressortissants maliens, car elle identifie l'ensemble des associations de migrants actives en 2011 intervenant dans les communes rurales de l'échantillon. En plus des associations basées en France, elle permet donc de caractériser le phénomène associatif des Maliens de l'extérieur résidant dans le reste du monde. Dans le module Commune de I'EMOP, nous dénombrons 794 associations de ressortissants basées à I'extérieur du Mali, dont 487, soit $61 \%$, en dehors de la France ${ }^{31}$. Comme nous l'avons fait pour les associations françaises, nous pouvons observer la répartition sur le territoire malien de l'activité de ces associations de migrants basées dans le reste du monde et la comparer aux chiffres disponibles sur l'émigration.

La répartition des flux d'émigration vers le reste du monde est bien plus homogène, entre les différentes régions du Mali, que la répartition des flux dirigés vers la France. Par souci de cohérence avec les données dont nous disposons sur le mouvement associatif (uniquement en milieu rural), le tableau 4 montre la répartition par région des migrants maliens originaires du milieu rural ${ }^{32} .19,7 \%$ d'entre eux proviennent de la région de Ségou, 19,4\% de la région de Koulikoro et $18,2 \%$ de la région de Mopti. Suivent les régions de Sikasso et Kayes dont proviennent respectivement $17,9 \%$ et $14,5 \%$ des émigrés

\footnotetext{
31 Les chiffres issus de l'enquête EMOP sont pondérés afin de corriger pour l'attrition dans I'échantillon. Le système de pondération adopté est présenté en annexe A.

32 Le tableau 7 en annexe $B$ donne la répartition de l'ensemble des émigrés maliens installés ailleurs qu'en France. La France est le deuxième pays d'accueil des émigrés maliens (17,2 \% d'entre eux y sont installés), après la Côte d'Ivoire qui en rassemble $39,3 \%$. Les autres destinations privilégiées des émigrés maliens sont principalement les pays d'Afrique de l'Ouest, puis le reste de I'Afrique.
} 
Tableau 3 : Les associations de migrants maliens, évolution dans le temps et répartition régionale

\begin{tabular}{|c|c|c|c|c|c|}
\hline & \multicolumn{5}{|c|}{$\begin{array}{l}\text { Associations de migrants maliens recensées au Journal Officiel } \\
\text { français }\end{array}$} \\
\hline & Total & $\begin{array}{l}\text { Déclarées } \\
\text { avant } 1995\end{array}$ & \begin{tabular}{|c|} 
Déclarées \\
entre 1995 \\
et 1999
\end{tabular} & $\begin{array}{c}\text { Déclarées } \\
\text { entre } 2000 \\
\text { et } 2004\end{array}$ & $\begin{array}{l}\text { Déclarées } \\
\text { depuis } 2005\end{array}$ \\
\hline Ensemble du Mali & 565 & 116 & 65 & 140 & 244 \\
\hline Milieu rural & $\begin{array}{l}431 \\
(76,3 \%)\end{array}$ & $\begin{array}{l}92 \\
(79,3 \%)\end{array}$ & $\begin{array}{l}43 \\
(66,2 \%)\end{array}$ & $\begin{array}{l}114 \\
(81,4 \%)\end{array}$ & $\begin{array}{l}182 \\
(74,6 \%)\end{array}$ \\
\hline Milieu urbain & \begin{tabular}{|l|}
64 \\
$(11,3 \%)$
\end{tabular} & \begin{tabular}{|l|}
14 \\
$(12,1 \%)$
\end{tabular} & $\begin{array}{l}11 \\
(16,9 \%)\end{array}$ & $\begin{array}{l}11 \\
(7,9 \%)\end{array}$ & $\begin{array}{l}28 \\
(11,5 \%)\end{array}$ \\
\hline \multicolumn{6}{|c|}{ Répartition par région (en \%) } \\
\hline Kayes & 68,1 & 70,7 & 75,4 & 70,7 & 63,5 \\
\hline Koulikoro & 11,9 & 11,2 & 7,7 & 12,9 & 12,7 \\
\hline Sikasso & 2,8 & 0,9 & 3,1 & 1,4 & 4,5 \\
\hline Ségou & 2,5 & 4,3 & 3,1 & 2,1 & 1,6 \\
\hline Mopti & 10,1 & 8,6 & 4,6 & 9,3 & 12,7 \\
\hline Tombouctou & 1,6 & 2,6 & 0 & 0,7 & 2 \\
\hline Gao & 0,9 & 0,9 & 3,1 & 0,7 & 0,4 \\
\hline Kidal & 0,5 & 0,9 & 3,1 & 0 & 0 \\
\hline Bamako & 1,6 & 0 & 1,5 & 1,4 & 2,5 \\
\hline \multicolumn{6}{|c|}{ Répartition par région, milieu rural (en \%) } \\
\hline Kayes & 72,6 & 76,1 & 74,4 & 76,3 & 68,1 \\
\hline Koulikoro & 11,4 & 9,8 & 9,3 & 12,3 & 12,1 \\
\hline Sikasso & 3,2 & 1,1 & 4,7 & 1,8 & 4,9 \\
\hline Ségou & 1,6 & 2,2 & 2,3 & 0,9 & 1,6 \\
\hline Mopti & 8,8 & 8,7 & 4,7 & 7 & 11 \\
\hline Tombouctou & 1,2 & 1,1 & 0 & 0,9 & 1,6 \\
\hline Gao & 0,7 & 1,1 & 0 & 0,9 & 0,5 \\
\hline Kidal & 0,5 & 0 & 4,7 & 0 & 0 \\
\hline
\end{tabular}

Source : Calculs des auteurs à partir des déclarations des associations de migrants maliens au Journal Officiel français, compilées sur www.refasso.com. 


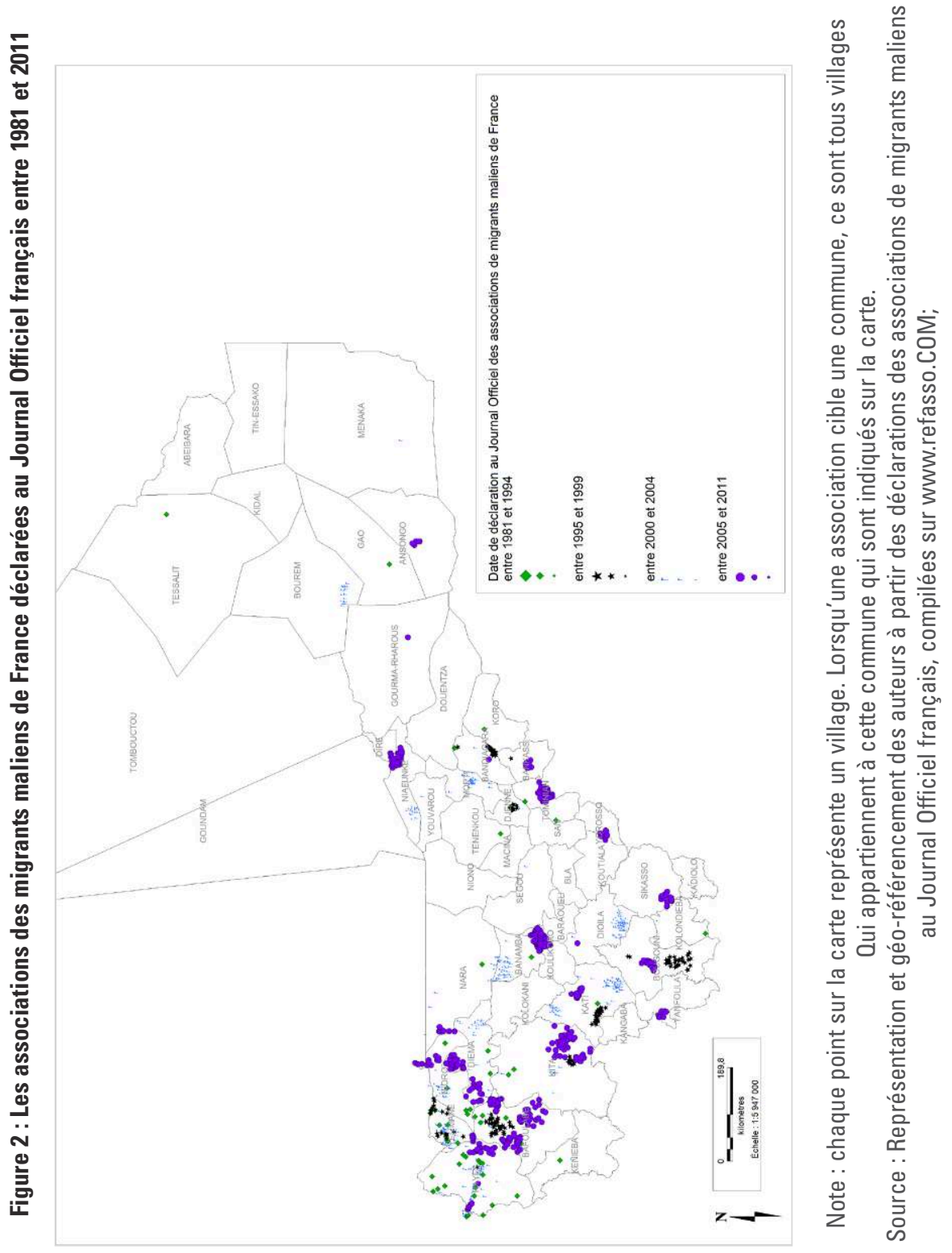


dans le reste du monde. Enfin, 7,5\% des émigrés maliens ruraux installés ailleurs qu'en France sont originaires de Tombouctou, 2,3\% de Gao et moins de $1 \%$ de la région de Kidal. D'après le module Commune de l'enquête EMOP, ce sont les régions de Mopti et de Koulikoro qui comptent le plus d'associations de ressortissants non françaises avec respectivement 133 associations (soit 27,3 \% des associations) et 127 associations (soit 26,1 \%). Suivent Kayes et Sikasso (102 et soixante-douze associations, respectivement $20,9 \%$ et $14,8 \%$ des associations), puis Ségou (vingt-sept associations, soit 5,5\% des associations) et enfin Gao et Tombouctou (respectivement quinze et onze associations, soit 3,1 \% et 2,3\% des associations). Notons que la région de Kidal n'en compte aucune d'après notre enquête. Si la prédominance des cinq régions du Sud est bien apparente, on ne peut statuer à partir de ces données sur la ville-région de Bamako puisque I'enquête EMOP ne concerne que les communes rurales.

La région de Mopti est donc la principale cible des associations de ressortissants maliens localisées à l'étranger hors France. Ainsi, l'importance respective des différentes régions maliennes dans la dynamique associative des ressortissants maliens de l'extérieur hors France ne reflète pas tout à fait la répartition des émigrés par région. La région de Ségou, première pourvoyeuse de migrants originaires du milieu rural vers le reste du monde (hors France), n'est que cinquième en termes d'activité associative des migrants. À l'inverse, les régions de Kayes, Koulikoro et Mopti sont caractérisées par une dynamique associative relativement forte par rapport au poids qu'elles représentent dans les flux d'émigration dirigés vers le reste du monde. Nous avions déjà fait ce constat pour les associations de migrants en France de la région de Mopti. Les régions de Koulikoro et de Kayes sont également les cibles d'une part importante des associations de migrants installés dans le reste du monde par rapport à leur contribution aux flux de départ tandis que les trois régions du Nord restent peu concernées par les phénomènes migratoire et associatif.

Tableau 4 : Répartition par région des stocks d'émigrés et des associations de ressortissants maliens originaires des zones rurales du Mali

\begin{tabular}{|l|l|l|l|l|}
\hline \multicolumn{1}{|c|}{ (en \%) } & $\begin{array}{c}\text { Part de la } \\
\text { population } \\
\text { émigrée en } \\
\text { France }\end{array}$ & $\begin{array}{c}\text { Part de la } \\
\text { population } \\
\text { émigrée dans le } \\
\text { reste du monde }\end{array}$ & $\begin{array}{c}\text { Part des } \\
\text { associations } \\
\text { basées en } \\
\text { France }\end{array}$ & $\begin{array}{c}\text { Part des } \\
\text { associations } \\
\text { basées dans le } \\
\text { reste du monde* }\end{array}$ \\
\hline Kayes & 77,4 & 14,5 & 57,3 & 20,9 \\
\hline Koulikoro & 11,3 & 19,4 & 28,3 & 26,1 \\
\hline Sikasso & 0 & 17,9 & 4,6 & 14,8 \\
\hline Ségou & 10,1 & 19,7 & 4,6 & 5,5 \\
\hline Mopti & 1,3 & 18,2 & 4,9 & 27,3 \\
\hline Tombouctou & 0 & 7,5 & 0 & 2,3 \\
\hline Gao & 0 & 2,3 & 0,03 & 3,1 \\
\hline Kidal & 0 & 0,6 & 0 & 0 \\
\hline
\end{tabular}

* Au sein de ces associations localisées dans le reste du monde, le nombre d'associations de migrants désagrégé par pays de résidence (et notamment en Côte d'Ivoire, la première destination des flux d'émigration malienne) n'est pas disponible.

Source : Calculs des auteurs à partir des données pondérées de l'enquête EMOP, modules GLAMMS, 2011, INSTAT IRD. Le système de pondération adopté est présenté en annexe $A$. 
En résumé, les régions les plus concernées par la dynamique associative des migrants, qu'ils soient installés en France ou dans le reste du monde, sont donc les cinq régions du Sud (Kayes, Koulikoro, Mopti, Sikasso, Ségou) alors que Tombouctou, Gao et Kidal sont peu ciblées par les associations de migrants. Nos deux sources de données apparaissent cohérentes l'une avec l'autre et nous informent sur l'intensité associative observée dans chacune des régions. Ce panorama des zones d'intervention des associations de ressortissants au Mali fait surtout bien ressortir les caractéristiques régionales du phénomène migratoire malien et la domination de la région de Kayes dans les flux de migrants dirigés vers la France. Concernant les autres régions maliennes, nos deux sources de données montrent que les Maliens originaires de la région de Mopti, qu'ils soient installés en France ou dans le reste du monde, ont une activité associative relativement forte par rapport à leur poids dans les flux d'émigration. À l'inverse, Bamako, dont partent beaucoup de migrants vers la France, est relativement peu ciblée par les associations de ressortissants ${ }^{33}$. Notre travail de collecte de données nous permet donc de montrer que les pratiques associatives des migrants maliens sont loin d'être un phénomène réservé aux migrations originaires de la région de Kayes et à destination de la France. Bien que ces flux aient indéniablement marqué I'histoire de la migration malienne et restent intenses (Pollet et Winter, 1971 ; Gubert, 2000 ; Daum, 1998 et 2000), la dynamique associative des migrants maliens est aujourd'hui un phénomène qui touche aussi d'autres régions du Mali et qui concerne également la diaspora malienne hors de France.

\section{Perspectives de recherche}

Le panorama du mouvement associatif des Maliens de l'extérieur qui vient d'être brossé incite à s'interroger sur les facteurs à l'origine des dynamiques spatio-temporelles observées. Leur répartition géographique soulève la question des spécificités des associations de migrants originaires des autres régions que Kayes et de ce qui les distingue des associations kayésiennes. Parmi elles, celles créées à l'initiative de migrants résidant en France ont-elles une insertion dans les réseaux de coopération décentralisée différente de celles créées dans d'autres pays de destination ? L'accélération de la dynamique associative observée depuis une quinzaine d'années soulève également les questions suivantes : en quoi la logique qui a présidé à la création des "nouvelles " associations se distingue-t-elle de la logique de création des premières caisses de solidarité villageoise? Les associations récentes se différencient-elles dans leurs modes de fonctionnement ou de financement, dans leurs choix d'activités ?

La constitution des deux bases de données présentées ici a finalement été motivée par notre souhait d'évaluer de manière quantitative le rôle des associations de migrants dans la fourniture en biens publics locaux des communes et villages maliens. Le fait de disposer de données sur l'ensemble du Mali permet d'apporter un éclairage intéressant à cette question. En effet, les travaux menés

33 Cette observation tirée du recensement des associations déclarées au Journal Officiel ne peut être ni infirmée ni confirmée par la seconde source de données dont nous disposons, le module Commune de l'enquête EMOP n'ayant été administré qu'en milieu rural. 
jusqu'à présent ont essentiellement adopté une approche qualitative de cette question et se sont portés sur des espaces géographiques limités, ceux de la région de Kayes ou de la vallée du fleuve Sénégal.

Une approche quantitative portant sur l'ensemble du Mali est rendue possible par les bases de données que nous avons constituées. Nous pouvons notamment examiner l'impact des associations de migrants sur le développement local, et plus particulièrement sur la fourniture de biens publics, non pas seulement dans la région de Kayes, mais aussi sur l'ensemble du territoire malien. Ainsi, d'après I'enquête EMOP, 3 \% des infrastructures publiques locales ${ }^{34}$ existant en 2011 dans les villages maliens ont bénéficié d'un financement des associations de migrants. Ce taux cache une concentration très forte des financements collectifs de migrants dans la région de Kayes. Dans cette région, en moyenne, presque une infrastructure sur cinq a bénéficié d'un financement total ou partiel de la part des migrants : c'est le cas de plus d'une école et d'une mosquée sur trois, de quasiment un centre de santé sur deux et de presque un point d'eau sur cinq (cf. Tableau 5 et Figure 3). Au niveau des communes, on constate que, depuis 2004, les associations de migrants ont participé au financement de 4,4\% des projets de développement des communes rurales sur l'ensemble du Mali. Ce taux s'élève à $13 \%$ dans la région de Kayes.

Tableau 5 : Part des infrastructures ayant bénéficié d'un financement des associations de migrants par région, 2011 (en pourcentage du total des infrastructures)

\begin{tabular}{|l|l|l|l|l|l|}
\hline \multicolumn{1}{|c|}{ (en \%) } & \multicolumn{1}{|c|}{ École } & \multicolumn{1}{c|}{$\begin{array}{c}\text { Centre de } \\
\text { santé }\end{array}$} & $\begin{array}{c}\text { Fontaine, } \\
\text { forage, puits }\end{array}$ & \multicolumn{1}{c|}{ Mosquée } & \multicolumn{1}{c|}{ Total } \\
\hline Mali & $\mathbf{6}$ & $\mathbf{8}$ & $\mathbf{3}$ & $\mathbf{1 2}$ & $\mathbf{3}$ \\
\hline Kayes & 34 & 42 & 19 & 32 & 19 \\
\hline Koulikoro & 4 & 7 & 0 & 4 & 0 \\
\hline Sikasso & 0 & 0 & 0 & 1 & 0 \\
\hline Ségou & 2 & 3 & 1 & 10 & 1 \\
\hline Mopti & 1 & 0 & 1 & 1 & 1 \\
\hline Nord & 3 & 3 & 3 & 9 & 3 \\
\hline
\end{tabular}

Source : Calculs des auteurs à partir d'EMOP, modules GLAMMS, 2011, INSTAT IRD.

Ces résultats peuvent être complétés à l'aide du recensement des associations de migrants déclarées au Journal Officiel français. Dans un article complémentaire à celui-ci (Chauvet et al., 2013), nous confrontons ce recensement aux données sur les infrastructures locales disponibles dans les Recensements Généraux de la Population et de I'Habitat (RGPH) réalisés par I'INSTAT du Mali en 1976, 1987, 1998 et 2009. En comparant les villages ciblés par une association de migrants recensée en France aux villages sans association, nous montrons ainsi que les villages dans lesquels intervient une association ont vu le nombre de leurs infrastructures publiques (écoles, centres de santé et bornes-fontaines, notamment) augmenter plus rapidement que les autres villages. Les quatre vagues de recensement des infrastructures nous permettent d'analyser l'impact des associations sur différentes sous-périodes. Il en ressort qu'elles sont davantage intervenues dans la construction d'écoles et de centres de santé dans

34 Écoles, centres de santé, points d'eau ou lieux de culte. 
les années 1980 et 1990, tandis que leur activité s'est concentrée sur l'approvisionnement en eau à partir de la fin des années $1990^{35}$.

Les villages ciblés par une association de migrants déclarée au Journal Officiel français ont donc vu certains types de leurs infrastructures augmenter plus vite que les autres villages sur la période étudiée. On aurait pu s'attendre à un tout autre résultat si un processus de réallocation de la dépense publique avait eu lieu en faveur des villages non " pris en charge " par des associations de migrants de France. Nos résultats suggèrent qu'un tel effet d'éviction ne s'est pas véritablement produit, et donc que les associations de migrants ont joué un rôle dans l'évolution des inégalités territoriales au niveau local. Des investigations plus poussées sont cependant nécessaires pour comprendre les mécanismes expliquant la complémentarité des investissements publics et privés collectifs. Les effets positifs de la présence d'associations de migrants de France proviennent-ils de la capacité des associations à obtenir des cofinancements de la part des communes françaises, des ONG ou d'autres agences de développement nationales et internationales ? En plus de ce levier financier, l'impact des associations de migrants transite-t-il par leur capacité à exercer un contrôle sur les autorités locales, à surveiller l'utilisation des fonds qu'elles envoient et à véhiculer des pratiques de bonne gouvernance?

C'est cette perspective que nous comptons explorer dans des recherches futures, en nous intéressant notamment à l'influence des associations de migrants sur la gouvernance locale au Mali. L'enquête EMOP est à cet égard riche en informations : elle apporte notamment des renseignements détaillés sur la composition du conseil municipal ainsi que sur les caractéristiques des conseillers municipaux. Aussi pourrons-nous voir dans quelle mesure la composition des conseils municipaux est différente dans les communes à forte intensité migratoire. Nous explorerons également la manière dont l'expérience migratoire des individus influence leur accès à des fonctions électives locales, pouvant ainsi modifier la composition des structures décisionnelles locales. Enfin, nous examinerons si la présence de migrants de retour dans les conseils municipaux modifie les choix d'allocations des biens publics.

Les dispositifs de collecte d'information sur le tissu associatif des migrants maliens présentés dans cet article renouvellent incontestablement nos connaissances. D'une part, nous confirmons les phénomènes déjà décrits, notamment par Lima (2005) et Gonin (2005), de propensions élevées des Kayésiens à migrer et à se regrouper en associations. D'autre part, nous montrons que cela n'est pas exclusif aux migrants de la région de Kayes : d'autres migrants en France ont créé des associations, notamment ceux originaires des régions de Koulikoro et de Mopti. Par ailleurs, les migrants installés dans d'autres pays ont aussi une activité associative vive.

Dans un premier temps, les bases de données constituées permettent d'analyser le rôle des associations de migrants dans le développement local du Mali. Dans un second temps, elles ont vocation à être mises à la disposition de la communauté des chercheurs comme des opérateurs du développement maliens, français et internationaux.

35 Pour plus de détails sur la méthodologie mise en œuvre et les résultats, voir Chauvet et al. (2013). 


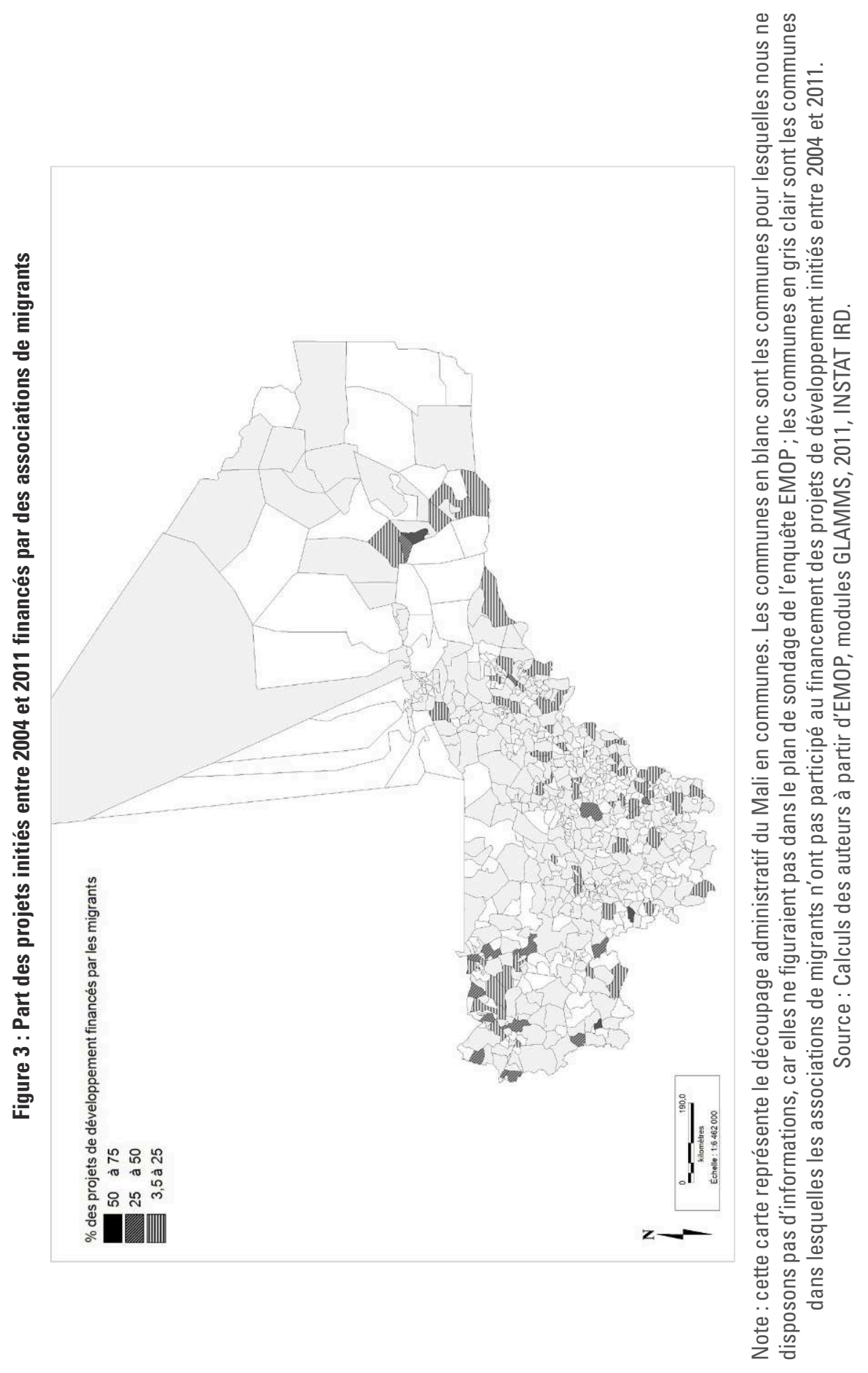




\section{Références bibliographiques}

Aparicio Francisco and Meseguer Covadonga (2008) Collective Remittances and the State: The 3x1 Program for Migrants in Mexico, Paper presented at the annual meeting of the APSA, Hynes Convention Center, Boston, Massachusetts, Aug 28, 2008.

Balique Hubert, Didierlaurent Monique et Marié Pierre (1983) Place des travailleurs migrants dans les processus de développement de la région de Kayes. Étude générale, Bamako/Paris, République du Mali, ministère de la Santé publique et des Affaires sociales/ministère des Relations extérieures (France).

Beauchemin Cris and Schoumaker Bruno (2009) Migrant Associations as Actors in Local Development? A National Event-History Analysis in Burkina Faso, World Development, 37 (12), pp. 1897-1913.

Charef Mohamed et Gonin Patrick (Éds.) (2005) Émigrés-immigrés dans le développement local, Agadir, Éditions Sud-Contact, $361 \mathrm{p}$.

Chauvet Lisa, Gubert Flore, Mercier Marion and Mesplé-Somps Sandrine (2013), Migrants' Home Town Associations and Local Development in Mali, Working paper, $n^{\circ}$ 2013/11, Paris, DIAL, 37 p.

Cisse Pierre et Daum Christophe (2009) Migrations internationales maliennes, recomposition des territoires migratoires et impacts sur les sociétés d'origine, in Document de synthèse des projets du programme FSP 2003-74 : migrations internationales, recompositions territoriales et développement, Paris, IRD, pp. 187-198.

Condé Julien et Diagne Pap Syr (1983) Les migrations internationales Sud-Nord: une étude de cas : les migrants maliens mauritaniens et sénégalais de la vallée du fleuve Sénégal en France, Paris, Organisation de Coopération et de Développement Économique, 292 p.

Daum Christophe (1993) Quand les immigrés construisent leur pays, Paris, L'Harmattan, 208p.

Daum Christophe (1998) Les associations maliennes en France, Migration, Développement et Citoyenneté, Paris, Karthala, 253 p.

Daum Christophe (1995) Les migrants, partenaires de la coopération internationale : le cas des Maliens de France, document de travail 107, Centre de Développement de I'OCDE, $44 \mathrm{p}$.

Daum Christophe (1994) Ici et là-bas, immigration et développement, Migrations Société, 32, pp. 99-110.

Daum Christophe, Diarra Hamedy, Gonin Patrick, Philippe Claire, Quiminal Catherine et Sylla Samba (1988) La fonction émigrée dans les stratégies de développement, Fontenay-aux-Roses-Paris, E.N.S. Fontenay-Saint-Cloud, Centre de Géographie Rurale-CNRS-ATP, 117 p.

Dia Hamidou (2008) Villages multi-situés du Fouta-Toro en France : le défi de la transition entre générations de caissiers, lettrés et citadins, Revue Asylon(s), 3, [en ligne]. URL : http://www.reseau-terra.eu/article713.html

Gauvrit Lisa et Le Bahers Goulven (2004) Pratiques associatives des migrants pour le développement de leur pays d'origine: le cas des migrants maliens de France originaires de la Région de Kayes, FSP Codéveloppement Mali, 110 p. 
Gonin Patrick (2005) Jeux d'acteurs et enjeux territoriaux : quelles migrations pour quel développement ? L'exemple du bassin du fleuve Sénégal (République du Mali), in Mohamed Charef et Patrick Gonin Éds., Émigrés-immigrés dans le développement local, Agadir, Éditions Sud-Contact, pp. 259-278.

Gonin Patrick (2001) Migrations développement : les utopies des années 90, in Réginald Blion et Isabelle Rigoni Éds, D'un voyage à l'autre. Des voix de l'immigration pour un développement pluriel, Paris, Karthala, pp. 25-48.

Gonin Patrick et Kotlok Nathalie (2009) Les associations de développement des migrants dans leurs pays d'origine, Accueillir, 252, pp. 12-16.

Gubert Flore (2000) Migration as a collective risk-coping strategy. Evidence from the Kayes area (Western Mali), Ph.D. thesis, University of Auvergne.

Iskander Natasha N. (2008) Diaspora Networks for National Infrastructure: Rural Morocco, 1985-2005, in Jennifer M. Brikenhoff, Diasporas and Development: Exploring the Potential, Washington, D. C., Lynne Rienner Publishers, pp. 163-183.

Kane Abdoulaye (2002) Senegal's village diaspora and those left ahead, in Deborah F. Brycesson and Ulla Vuorella, The transnational family, Oxford-New York, Bloomsbury Academic, pp. 245-263.

Lacroix Thomas (2005) Les réseaux marocains du développement, géographie du transnational, politiques du territorial, Paris, Presses de la Fondation Nationale des Sciences Politiques, 257 p.

Lacroix Thomas (2009) Migration, Développement, Codéveloppement : quels acteurs pour quels discours ?, Rapport de synthèse, Paris, Institut Panos, 55 p.

Lacroix Thomas (2006) Les étrangers de la coopération, Projet, hors-série, pp. 72-77.

Lacroix Thomas, Sall Leyla et Salzbrunn Monika (2008) Marocains et Sénégalais de France : permanences et évolution des relations transnationales, Revue Européenne des Migrations Internationales, 24 (2), pp. 23-43.

Lima Stéphanie (2005) Les espaces associatifs face aux communes rurales. Recompositions spatiales, émergence des acteurs locaux et nouvelles perspectives pour le déveleppement dans la région de Kayes (Mali), in Mohamed Charef et Patrick Gonin Eds., Emigrés-immigrés dans le développement local, Agadir, Éditions Sud-Contact, pp. 279-309.

Navarra Cecilia et Salis Ester (2011) L'associationnisme sénégalais en Italie : une revue de la littérature, Working paper, $\mathrm{n}^{\circ}$ 2011/02, Paris, DIAL, $36 \mathrm{p}$.

Orozco Manuel and Welle Kate (2006) Hometown Associations and Development: Ownership, Correspondence, Sustainability and Replicability, in Barbara J. Merz, New Patterns for Mexico: Observations on Remittances, Philanthropic Giving, and Equitable Development, Cambridge, Harvard University, pp. 157-179.

Pollet Eric et Winter Grace (1971) La Société soninké (Dyahunu, Mali), Université de Bruxelles, 566 p.

Quiminal Catherine (2006) Décentralisation, démocratie et migrations dans la région de Kayes, in Claure Fay, Yaouaga F. Koné et Catherine Quiminal, Décentralisation et pouvoirs en Afrique : En contrepoint, modèles territoriaux français, Paris, IRD Editions, pp. 239-262. 
Quiminal Catherine (1994) Le rôle des immigrés dans les projets de développement et les formes de coopération possibles dans la vallée du fleuve Sénégal, in Migration et Développement : un nouveau partenariat pour la coopération, Paris, OCDE, pp. 329-336.

Quiminal Catherine (1993) Le rôle des immigrés dans les projets de développement et les formes de coopération possibles à moyen terme dans la région du fleuve Sénégal, présentation pour la conférence "Migrations et coopération internationale : les enjeux pour les pays de I'OCDE ", Madrid.

Quiminal Catherine (1991) Gens d'ici et d'ailleurs, Migrations soninké et transformations villageoises, Paris, Christian Bourgeois, 224 p.

Riccio Bruno (2007) Associationnisme, capital social et potentialités de co-développement parmi les migrants sénégalais de la province de Bergame, in Sebastiano Ceschi et Andrea Stocchiero, Relations transnationales et co-développement. Associations et entrepreneurs sénégalais entre Italie et lieux d'origine, Paris, L'Harmattan, $271 \mathrm{p}$.

Simpser Alberto (2010) Do Remittances Complement or Substitute GovernmentProvided Public Goods? A Study of the 3x1 Program in Mexico, Paper presented at the 67th annual meeting of the Midwest Political Science Association, The Palmer House Hilton, Chicago, IL.

Tandian Aly (2005) Stratégies d'autonomie, investissements développementalistes et besoins collectifs, nouvelles figures de migrants de la vallée du fleuve Sénégal, in Mohamed Charef et Patrick Gonin Éds., Émigrés-immigrés dans le développement local, Agadir, Éditions Sud-Contact, pp. 233-255. 


\section{Annexes}

\section{Annexe A : Correction des poids de l'enquête EMOP du fait de l'attrition de l'échantillon des communes rurales}

L'enquête EMOP a été conçue pour être représentative aux niveaux national et régional. Cependant, soixante questionnaires du module Commune, sur un échantillon initial représentatif de 431 communes, n'ont pas été administrés pour des raisons qui nous sont inconnues. La disparition de ces communes génère un biais potentiel dans l'échantillonnage que nous corrigeons grâce à un système de pondération.

La pondération assure que les communes présentes dans le module Commune soient représentatives de la répartition régionale de la population présente dans l'échantillon initial. Le tableau 6 présente la répartition par région malienne des communes, des communes enquêtées, de la population de l'échantillon EMOP initial et de la population enquêtée par le module Commune. Il nous permet de visualiser la représentativité de l'échantillon du module Commune d'EMOP.

Le poids alloué à chaque région malienne est égal au rapport entre la part de la population de la région dans l'échantillon initial représentatif (colonne 2) et la part de sa population dans l'échantillon final (colonne 4).

Ce mode de correction de l'attrition de l'échantillon n'est valide que si les communes manquantes ont les mêmes caractéristiques, en moyenne, que les communes de l'échantillon initial. Les tests statistiques que nous avons menés confirment la similitude entre ces deux sous-groupes, validant notre méthode de pondération (ces tests ne sont pas reproduits dans cet article, mais sont disponibles auprès des auteurs).

Tableau 6 : Représentativité du module Commune

\begin{tabular}{|l|l|l|l|l|}
\hline & \multicolumn{1}{|c|}{ (1) } & \multicolumn{1}{|c|}{ (2) } & \multicolumn{1}{c|}{ (3) } & \multicolumn{1}{|c|}{ (4) } \\
\cline { 2 - 5 } & \multicolumn{1}{|c|}{$\begin{array}{c}\text { Pombre de } \\
\text { RGPH (2009) }\end{array}$} & $\begin{array}{c}\text { Part de la } \\
\text { population - } \\
\text { Échantillon } \\
\text { initial EMOP }\end{array}$ & $\begin{array}{c}\text { Nombre de } \\
\text { communes } \\
\text { enquêtées - } \\
\text { EMOP }\end{array}$ & $\begin{array}{c}\text { Part de la } \\
\text { population - } \\
\text { EMOP Commune }\end{array}$ \\
\hline Kayes & 129 & $14,7 \%$ & 62 & $13,3 \%$ \\
\hline Koulikoro & 108 & $19,4 \%$ & 50 & $16,8 \%$ \\
\hline Sikasso & 147 & $17,8 \%$ & 65 & $18 \%$ \\
\hline Ségou & 118 & $19,5 \%$ & 77 & $20,7 \%$ \\
\hline Mopti & 108 & $18,2 \%$ & 75 & $20,3 \%$ \\
\hline Tombouctou & 52 & $7,5 \%$ & 25 & $7,9 \%$ \\
\hline Gao & 24 & $2,3 \%$ & 12 & $2,6 \%$ \\
\hline Kidal & 11 & $0,6 \%$ & 5 & $0,5 \%$ \\
\hline
\end{tabular}

Source : Calculs des auteurs à partir des données de l'enquête EMOP, modules GLAMMS, 2011, INSTAT IRD. Les données de la colonne 1 sont issues du Recensement Général de la Population (RGPH, 2009). 


\section{Annexe B}

Tableau 7 : L'émigration malienne en 2011

\begin{tabular}{|c|c|c|c|c|c|c|c|c|}
\hline & 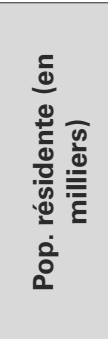 & 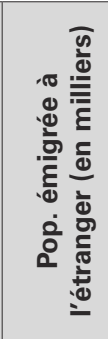 & 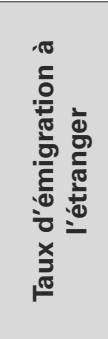 & 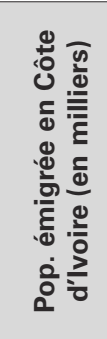 & 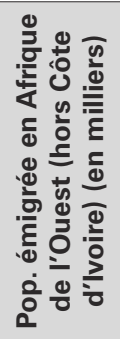 & 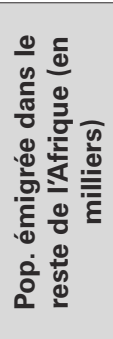 & 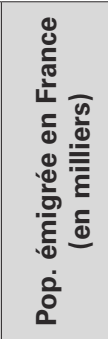 & 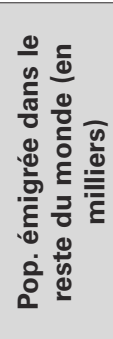 \\
\hline Mali & 16119 & 327,9 & $1,99 \%$ & \begin{tabular}{|l|}
126 \\
$(39 \%)$
\end{tabular} & \begin{tabular}{|l|}
47 \\
$(14 \%)$
\end{tabular} & $\begin{array}{l}64 \\
(19 \%)\end{array}$ & $\begin{array}{l}55 \\
(17 \%)\end{array}$ & \begin{tabular}{|l|}
28 \\
$(9 \%)$ \\
\end{tabular} \\
\hline Kayes & 2301 & 89,6 & $3,75 \%$ & 7 & 17 & 22 & 33 & 8 \\
\hline Koulikoro & 2587 & 37,9 & $1,44 \%$ & 11 & 1 & 10 & 8 & 6 \\
\hline Sikasso & 2827 & 74,2 & $2,56 \%$ & 57 & 6 & 8 & 1 & 1 \\
\hline Ségou & 2501 & 32,9 & $1,30 \%$ & 13 & 4 & 8 & 4 & 5 \\
\hline Mopti & 2291 & 42,6 & $1,83 \%$ & 23 & 5 & 7 & 0 & 6 \\
\hline $\begin{array}{l}\text { Tombouctou, } \\
\text { Gao et Kidal }\end{array}$ & 1571 & 24,3 & $1,52 \%$ & 7 & 13 & 3 & 0 & 1 \\
\hline Bamako & 2042 & 26,4 & $1,28 \%$ & 8 & 2 & 5 & 8 & 2 \\
\hline \multicolumn{9}{|c|}{ Répartition par région (en $\%$ du total) } \\
\hline Mali & 100 & 100 & & 100 & 100 & 100 & 100 & 100 \\
\hline Kayes & 14 & 27 & & 5 & 35,6 & 35 & 60 & 27 \\
\hline Koulikoro & 16 & 12 & & 9 & 3,1 & 16 & 15 & 23 \\
\hline Sikasso & 18 & 23 & & 45 & 11,9 & 12 & 2 & 3 \\
\hline Ségou & 16 & 10 & & 10 & 7,5 & 13 & 6 & 16 \\
\hline Mopti & 14 & 13 & & 18 & 11,5 & 12 & 1 & 20 \\
\hline $\begin{array}{l}\text { Tombouctou, } \\
\text { Gao et Kidal }\end{array}$ & 10 & 7 & & 6 & 27,0 & 5 & 0 & 2 \\
\hline Bamako & 13 & 8 & & 6 & 3,4 & 9 & 15 & 8 \\
\hline
\end{tabular}

Source : Calculs des auteurs à partir d’EMOP, modules GLAMMS, 2011, INSTAT IRD. 


\section{Claire Bernard, Lisa Chauvet, Flore Gubert, Marion Mercier et Sandrine Mesplé-Somps}

\section{La dynamique associative des Maliens de l'extérieur : enseignements tirés de deux dispositifs d'enquête originaux}

Cet article présente deux dispositifs originaux de collecte de données sur les associations de migrants maliens présentes en France et dans le reste du monde : le premier s'est attaché à recenser les associations de migrants maliens déclarées en France et à identifier leurs terrains d'intervention au Mali. Le second s'est greffé à une enquête nationale représentative, réalisée en 2011 au Mali, et a consisté en l'ajout d'un module recensant l'ensemble des associations de ressortissants intervenant dans les communes et villages enquêtés. Ces deux dispositifs complémentaires confirment la propension élevée des Maliens de la région de Kayes à migrer et à se regrouper en associations. Ils montrent également que ce fort engagement associatif n'est pas réservé aux migrants originaires de la région de Kayes et résidant en France : les Maliens de France originaires d'autres régions, notamment Koulikoro et Mopti, sont eux aussi à l'origine de la création de nombreuses associations, tout comme les Maliens résidant dans d'autres pays.

\section{Malian Migrants' Home Town Associations: Insights from Two Original Surveys}

This article presents two original datasets on Malian Migrants' Home Town Associations (HTAs) located in France and in the rest of the world. The first is a census of the HTAs declared in the French Journal Officiel that provides detailed information on their area of intervention in Mali. The second is a representative survey implemented in Mali in 2011 that collected exhaustive information on abroad-based HTAs intervening in each commune and village of the sample. These two complementary datasets confirm that Malians coming from the region of Kayes are prone to gather in HTAs, notably in France. They also show that this propensity to create HTAs is not exclusive to these migrants. A lot of HTAs were created by Malians coming from the regions of Koulikoro and Mopti, in France as well as in other destination countries.

\section{La dinámica asociativa de los malienses del exterior: datos extraídos de dos dispositivos de encuesta originales}

Este artículo presenta dos dispositivos originales de recogida de datos sobre las asociaciones de emigrantes malienses presentes en Francia y en el resto del mundo: el primero se dedicó a censar las asociaciones de emigrantes malienses declaradas en Francia y a identificar sus ámbitos de intervención en Mali. El segundo se incorporó a una encuesta nacional representativa, realizada en 2011 en Mali, y consistió en el añadido de un módulo que censaba el conjunto de las asociaciones de nacionales que intervenía en los municipios y los pueblos investigados. Estos dos dispositivos complementarios confirman la propensión elevada de los malienses de la región de Kayes a emigrar y a reagruparse en asociaciones. También muestran que este compromiso fuerte y asociativo no está reservado a los emigrantes originarios de la región de Kayes y que residen en Francia: los malienses de Francia originarios de otras regiones, particularmente Koulikoro y Mopti, están también en el origen de la creación de numerosas asociaciones, del mismo modo que los malienses residentes en otros países. 